\title{
Properdin Is a Modulator of Tumour Immunity in a Syngeneic Mouse Melanoma Model
}

\author{
Izzat A. M. Al-Rayahi ${ }^{1,2}$, Lee R. Machado ${ }^{3, * \mathbb{D}}$ and Cordula M. Stover ${ }^{1} \mathbb{D}$ \\ 1 Department of Respiratory Sciences, University of Leicester, Leicester LE1 9HN, UK; \\ izzatalrayahi@gmail.com (I.A.M.A.-R.); cms13@le.ac.uk (C.M.S.) \\ 2 Department of Medical Laboratory Technology, College of Health and Medical Technology, \\ Middle Technical University, Baghdad 10047, Iraq \\ 3 Centre for Physical Activity and Life Sciences, University of Northampton, Northampton NN1 5PH, UK \\ * Correspondence: Lee.Machado@northampton.ac.uk
}

Citation: Al-Rayahi, I.A.M.; Machado, L.R.; Stover, C.M. Properdin Is a Modulator of Tumour Immunity in a Syngeneic Mouse Melanoma Model. Medicina 2021, 57, 85. https://doi.org/10.3390/ medicina57020085

Academic Editor: Māra Pilmane

Received: 14 December 2020

Accepted: 16 January 2021

Published: 21 January 2021

Publisher's Note: MDPI stays neutral with regard to jurisdictional claims in published maps and institutional affiliations.

Copyright: (c) 2021 by the authors. Licensee MDPI, Basel, Switzerland. This article is an open access article distributed under the terms and conditions of the Creative Commons Attribution (CC BY) license (https:// creativecommons.org/licenses/by/ $4.0 /)$.

\begin{abstract}
Background and Objectives: Tumours are often low immunogenic. The role of complement, an innate immune defence system, in tumour control has begun to be elucidated, but findings are conflicting. A role for properdin, an amplifier of complement activation, in tumour control has recently been implicated. Materials and Methods: Properdin-deficient and congenic wildtype mice were injected subcutaneously with B16F10 melanoma cells. Tumour mass and chemokine profile were assessed. The frequencies of $\mathrm{CD} 45^{+} \mathrm{CD} 11 \mathrm{~b}^{+} \mathrm{Gr}-1^{+}$cells were determined from tumours and spleens, and $\mathrm{CD} 206^{+} \mathrm{F} 4 / 80^{+}$cells were evaluated in spleens. Sera were analysed for $\mathrm{C} 5 \mathrm{a}$, sC5b-9, and CCL2. Results: Whilst there was no difference in tumour growth at study endpoint, properdin-deficient mice had significantly fewer myeloid-derived suppressor cells (MDSCs) in their tumours and spleens. Splenic M2 type macrophages and serum levels of C5a, sC5b-9, and CCL2 were decreased in properdin-deficient compared to wildtype mice. Conclusions: The presence of intact complement amplification sustains an environment that lessens potential anti-tumour responses.
\end{abstract}

Keywords: CCL2; MDSC; melanoma; B16

\section{Introduction}

Mouse models of various tumours have been employed as preclinical models to inform or direct management of human disease, though they have their limitations [1]. Syngeneic tumour models involve implantation of tumour cells of the same genetic background as the host and therefore mimic the human situation when tumours arise in an immunocompetent host after having escaped immune recognition [2]. B16 melanoma models are poorly immunogenic [3]. In comparison to highly immunogenic solid tumour models, B16induced tumours show relatively greater expansion and a paucity in expression of genes characteristic of activated T cells and dendritic cells. Immune suppressive cell populations (regulatory T cells and myeloid-derived suppressor cells (MDSCs)) are detectable in tumour as well as spleen and associate with a poor response to experimental immunotherapy [4]. The mouse spleen is able to receive progenitor cells from bone marrow (in the adult) and, unlike human, provides a suitable niche for hematopoietic differentiation [5], from where they may seed target tissues including tumours [6]. Monocytic replenishment from spleen in a mouse model of lung adenocarcinoma was CCR2 dependent and impacted on tumour growth [7].

Recent studies have highlighted the importance of innate immune pathways in modulating the tumour microenvironment. Specifically, complement anaphylatoxins lead to activation, movement, and proliferation of cells. C5a and C3a bind to MDSCs, tumour infiltrating lymphocytes, and tumour cells, and thereby dampen adaptive immunity and consequently increase metastatic potential [8]. In a syngeneic model of cervical cancer, primary tumours in the flanks showed $\mathrm{C} 3$ dependent tumour growth, activation of the 
classical pathway of complement, and C5a mediated recruitment of $C D 45^{+} \mathrm{CD} 11 \mathrm{~b}^{\text {+hi }} \mathrm{Gr} 1^{+}$ MDSC into tumours [9].

B16F10 is a melanoma cell line which is injected subcutaneously and allows investigation of an orthotopic tumour (in the skin). A 14-day model of B16F10 melanoma growth using wildtype and $\mathrm{C}^{-/-}$mice showed that smaller tumour development was associated with greater IL-10 expression in $\mathrm{C}^{-/-} \mathrm{CD}^{+} \mathrm{T}$ cells, which were increased, while the numbers of $\mathrm{CD}_{4} 5^{+} \mathrm{CD} 11 \mathrm{~b}^{+} \mathrm{Gr}^{+}$tumour infiltrating leukocytes remained comparable [10].

Similarly, a C3aR-positive host environment was found to benefit tumour growth in another 14-day model of B16F10 melanoma comparing wildtype and $\mathrm{C}^{2} \mathrm{R}^{-/}$- mice. $\mathrm{C}_{3} \mathrm{R}^{-1-}$ mice showed a change in the proportions of different leukocyte subpopulations in tumour infiltrates and had increased CCL5 in lysates from tumours. Daily intraperitoneal injections with $\mathrm{C} 5 \mathrm{a} R$ or $\mathrm{C} 3 \mathrm{aR}$ antagonists of tumour-bearing mice led to a significant reduction in the tumour mass compared to the vehicle controls [11]. In contrast, a dependence on $\mathrm{C} 3$ or $\mathrm{C} 5$ could not be demonstrated in a different study of B16F10 melanoma growth over 14 days but showed a significant contribution of $\mathrm{C} 1 \mathrm{q}$ to tumour development [12]. These differences may point to differences in cell culture conditions or in a subline that has evolved, particularly as metastatic potential was evaluated in the latter but not the two former studies.

Properdin is a positive regulator of the alternative pathway of complement activation that stabilises the alternative pathway convertases which generate C3a and C5a. We have previously studied the in vitro effect of B16F10 conditioned medium on the profile of bone marrow-derived macrophages of properdin-deficient and wildtype mice. We found that macrophages from properdin-deficient mice were skewed towards an M2 phenotype and speculated that "properdin insufficiency may promote a tumour environment that helps the tumour evade the immune response" [13].

Our aim was to determine whether properdin is an important modulator of tumour growth in vivo. We found that while overall tumour growth was not significantly impacted by the absence of properdin, there was significantly less CCL2 chemokine and fewer Gr1 ${ }^{+}$ $\mathrm{CD}_{11 b^{+}}$MDSCs as well as M2 type macrophages compared with wildtype counterparts.

\section{Materials and Methods}

\subsection{Mice}

All animal experiments were carried out after acquiring approval from the institutional ethical review committee (University of Leicester) and conducted in accordance with United Kingdom Home Office regulations (project license PPL 80/2354-approved 1 December 2014). The severity of the protocol used in this paper was classed as moderate. Age-matched male congenic mice were taken from the properdin-deficient mouse colony held at the University of Leicester [14]. Wildtype and properdin-deficient mice were subcutaneously injected with tumour cell suspensions in their flanks and monitored for their wellbeing and tumour growth every other day to daily. Some received an additional injection of luciferin where indicated (imaging). Mice reached their endpoint (tumour size: $1 \mathrm{~cm}$ in one dimension) within 14 days.

\subsection{Cells}

Mouse melanoma cells B16F10 were grown as described [13] and extensively washed in phosphate buffered saline (PBS) prior to injection at $1.6 \times 10^{5}$ cells $/ 100 \mu \mathrm{L}$. B16F10-luc cells (mouse melanoma cells expressing luciferase) were kindly provided by Dr. Victoria Brentville (Scancell, Nottingham, UK) and injected at a dose of $4 \times 10^{5}$ cells $/ 100 \mu \mathrm{L}$. Cells were confirmed to be mycoplasma negative using the EZ-PCR Mycoplasma Test Kit (Biological Industries, Cromwell, CT, USA).

\subsection{Determination of Tumour Mass, Bioimaging}

Callipers were used to measure baseline tumour width and length $\left(\mathrm{mm}^{3}\right)$. The following formula was used to calculate tumour volume because of the ellipsoid shape of 
the tumour mass: $0.5 \times A \times B^{2}$, where $A$ and $B$ are the diameters in $\mathrm{mm}$. In other groups, tumours were weighed after removal at necropsy. Bioluminescence was used in others. To do this, mice which had received B16F10-luc cells were injected in the scruff of their neck with $150 \mu \mathrm{g} / \mathrm{kg}$ luciferin subcutaneously and their tumours were imaged using IVIS ${ }^{\circledR}$ Spectrum In Vivo Imaging System (Caliper Life Sciences, Hopkinton, MA, USA) a pilot experiment to discern distribution kinetics of luciferin. Light emission was measured under anaesthesia with recovery $10 \mathrm{~min}$ after substrate injection.

\subsection{Proteome Array Analysis}

Tumour lysates were prepared and membranes (Proteome Profiler ${ }^{\mathrm{TM}}$ Array; R\&D Systems, Abingdon, UK) were hybridised with $300 \mu \mathrm{g}$ total protein. Pixel densities on developed X-ray film were analysed using a transmission mode scanner and image analysis software. A template was created to analyse the pixel density of each spot on the array. Then signal values were exported to an Excel (Microsoft Excel 2013) spreadsheet for analysis. The average signal of the pair of duplicate spots representing each cytokine was determined. The signal from a clear area of the array or negative control spots was used as a background value, and the background signal was subtracted from each spot.

\subsection{ELISA}

ELISA sandwich assays were used to detect C5a (R\&D Systems, DY2150), CCL2 (Affymetrix eBioscience, 88-7391, Fisher Scientific Loughborough, UK), and C5b-9 (MyBioSource, MBS703522, San Diego, CA, USA) according to the manufacturers' instructions.

\subsection{Flow Cytometry}

For flow cytometry, $1 \times 10^{6}$ cell $/ \mathrm{mL}$ were resuspended in PBS supplemented with $1 \%$ FBS. The cells were incubated with purified anti-Fc receptor blocking antibody (antiCD16/CD32 from Biolegend, San Diego, CA, UK) for $30 \mathrm{~min}$ in ice before staining with saturating concentrations of antibodies specific for CD45 (APC, clone RA3-6B2; eBioscience), CD11b (PE, clone M1/70; Ebioscience), Gr-1 (FITC, clone RB6-8C5; eBioscience), F4/80 (FITC, clone BM8, 1:250; Biolegend), or CD206 (APC, clone C068C2, 1:250; Biolegend). Cells were washed and analysed using either CellQuest or FACSDiva 6.0 software (BD Biosciences, San Jose, CA, USA).

\subsection{Statistical Analysis}

Statistical analysis was performed using GraphPad v8.0.1 (GraphPad Software) using unpaired $t$-test. Serum and single cell suspensions were analysed from three independent experiments. ELISA for CCL2, C5b-9, and C5a in sera and flow cytometry results for cell phenotyping were performed on identical mice.

\section{Results}

\subsection{Properdin-Deficient and Wildtype Mice Have Comparable Tumour Load}

A pilot study determined subcutaneous, non-infiltrative, and non-metastasising growth. The tumour was non-irritant and innocuous. The endpoint (diameter of tumour $1 \mathrm{~cm}$ ) was reached between 12 and 14 days. Subsequently, properdin-deficient and congenic wildtype mice were implanted subcutaneously with the B16F10 melanoma cell line. Tumours were measured by callipers and the volume was calculated using the formula for ellipsoid shapes (Figure 1a). As tumours were often uneven in their circumferences, these measurements approximated the real mass. Therefore, tumours were excised and weighed (Figure $1 b$ ). In both cases, there was a bimodal distribution, with 3/11 tumours being much larger than the rest and 5/14 wildtype mice clearly having large tumour weights. Properdin-deficient mice had much less variable growth of tumour mass. Statistically, there was no difference between the groups. For in vivo assessment of the tumour burden, we used the B16-Luc line which expresses luciferase. The tumour mass was captured by bioluminescent imaging (Figure 1c). The distribution in average radiance was 
widespread; there was no significant difference in derived tumour mass between wild type and properdin-deficient mice. One caveat is that necrotic areas alter luminescence intensity, so tumour mass seemed to be the more reliable measurement.

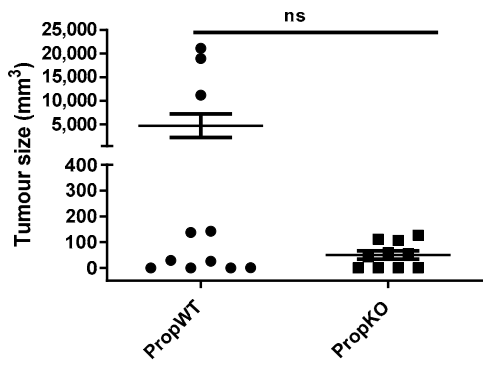

(a)

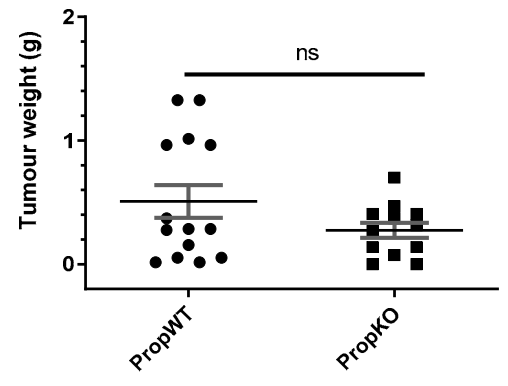

(b)

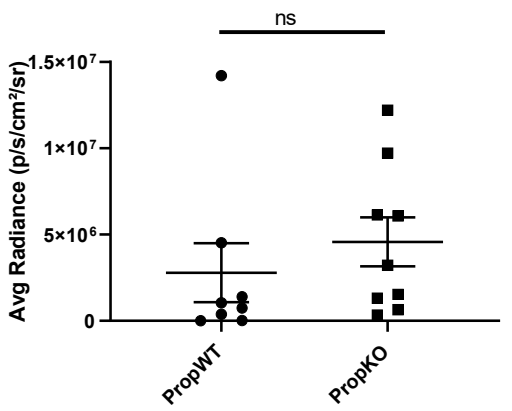

(c)

Figure 1. Tumour burden in properdin-deficient and wildtype mice. Wildtype (PropWT) and properdin-deficient (PropKO) mice were injected subcutaneously with B16F10 cells. After 14 days, the tumour size was measured by callipers. Values are expressed as mean \pm SEM from two independent experiments (a). The weight of tumours was established from three independent experiments (including the ones described in a) and values are expressed as mean \pm SEM (b). Quantitative analysis of the luciferase signal emitted from the tumour after injection of substrate 12 days following subcutaneous injection of B16F10-luc cells. Values are expressed as mean \pm SEM from two independent experiments (c). Data were analysed by two-tailed Mann-Whitney test.

\subsection{Inflammatory Marker Expression in B16 Tumours is Altered in Properdin-Deficient Mice}

Tumours extracted from a subgroup of mice matched for the size of tumours were analysed quantitatively for the presence of important mediators. Of forty mediators evaluated, only TIMP1, IL-13, CCL2, and CCL3 were significantly different between the tumour lysates prepared from the two genotypes, where properdin-deficient mice showed reductions in the chemoattractants CCL2, CCL3, the Th2 cytokine IL-13, and the metalloproteinase inhibitor TIMP-1 (Table 1 and Supplementary Figure S2).

Table 1. Identification of genotype-discriminatory mediators by Mouse Cytokine Array in tumour lysates of wildtype (PropWT) and properdin-deficient (PropKO) mice.

\begin{tabular}{cccc}
\hline & PropWT $(\boldsymbol{n}=\mathbf{3})$ & PropKO $(\boldsymbol{n}=\mathbf{3})$ & $p$-Value \\
\hline TIMP1 & $45.72 \pm 4.19$ & $10.41 \pm 0.91$ & $<0.002$ \\
IL-13 & $5.24 \pm 1.36$ & $0.88 \pm 0.35$ & $<0.05$ \\
CCL2 & $97.49 \pm 1.11$ & $1.37 \pm 0.17$ & $<0.0001$ \\
CCL3 & $46.31 \pm 3.01$ & $0.93 \pm 0.41$ & $<0.0002$
\end{tabular}

Three tumours of comparable sizes from each genotype were pooled, and extracted protein was analysed using Mouse Cytokine Array (R\&D Systems). Reactivities were quantified densitometrically. Data are expressed as means $\pm S D$ and were analysed by unpaired two-tailed $t$-test.

\subsection{Tumour-Bearing Properdin-Deficient Mice Have Dysregulated Systemic and Tumour Associated Responses}

C5a, sCb-9, and CCL2 were measured at endpoint of the study (14 days) in the sera of tumour-bearing properdin-deficient and wildtype and were found to be significantly decreased in the deficient animals (Figure 2). 


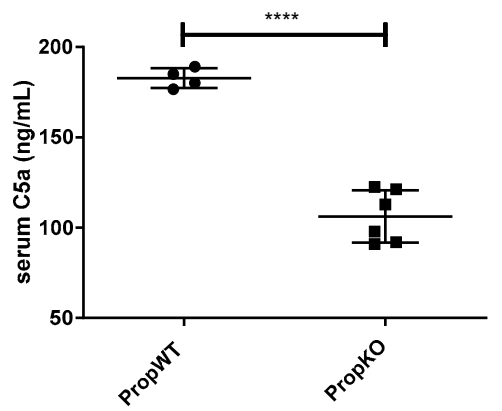

(a)

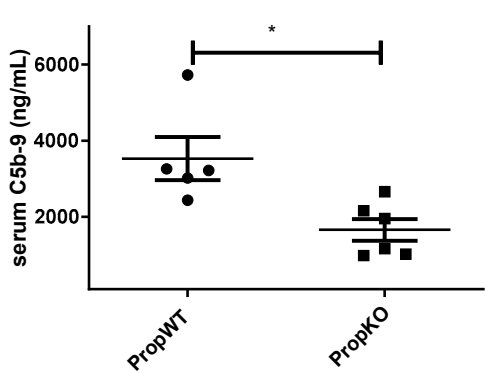

(b)

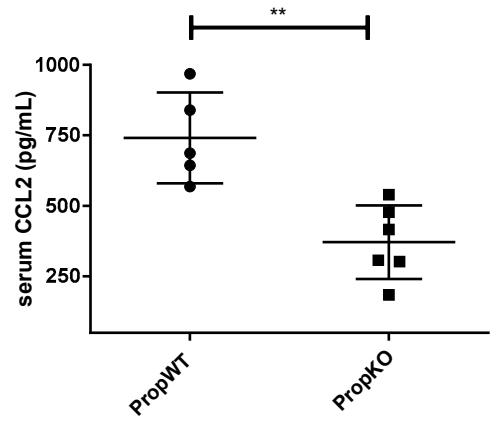

(c)

Figure 2. Serum levels of properdin- deficient (PropKO) and wildtype (PropWT) tumour-bearing mice for levels of C5a (a), C5b-9 (b), and CCL2 (c). Data are presented as means \pm SEM. Two tailed $t$-tests were performed, ${ }^{*} p<0.05,{ }^{* *} p<0.01$, **** $p<0.0001$.

We next examined whether the reduction of these immune mediators in serum would mirror alterations in MDSC and M2 type macrophages of properdin-deficient mice from wildtype mice. Single cell suspensions were prepared from spleens and tumours of experimental mice 14 days after subcutaneous injection of B16F10 cells in their flanks. We first examined the frequency of MDSCs in the tumours of properdin-deficient and wildtype mice by staining with lineage-specific antibodies (Supplementary Figure S1). Noticeably, properdin-deficient mice had fewer MDSCs (CD45, Gr-1, and CD11b) compared with wildtype mice (Figure 3a). The lower frequency of MDSCs in the tumours of properdin-deficient mice compared to wildtype animals was mirrored in the splenocyte population, where MDSCs and M2 macrophage numbers were reduced in tumour bearing properdin-deficient animals. Tumour-bearing properdin wildtype mice had a higher number of $\mathrm{CD}_{20} 6^{+}$ $\mathrm{F} 4 / 80^{+}$macrophages in the spleen compared with tumour-bearing properdin-deficient mice (Figure 3b,c).

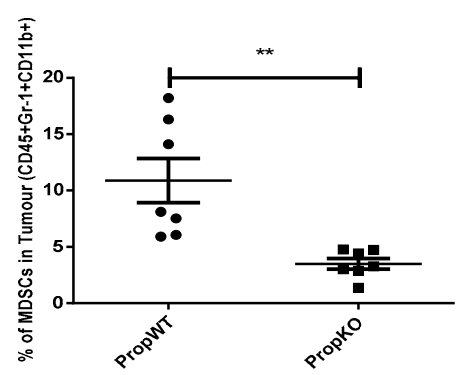

(a)

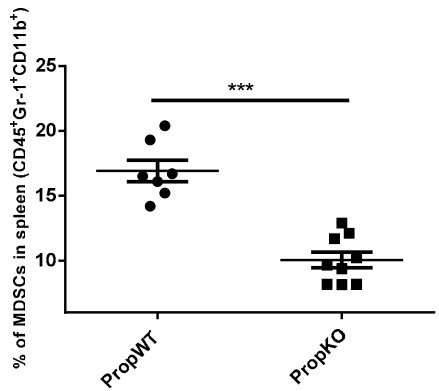

(b)

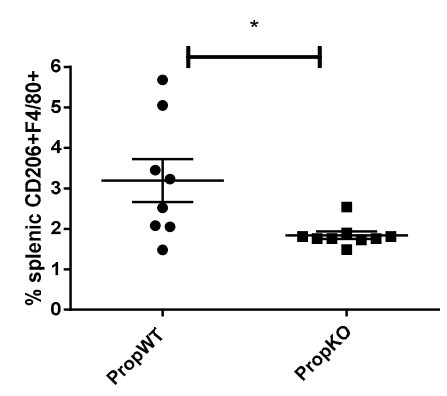

(c)

Figure 3. $\mathrm{CD} 45^{+} \mathrm{CD} 11 \mathrm{~b}^{+} \mathrm{Gr}-1^{+}$population in tumours and spleens and $\mathrm{CD} 206^{+} \mathrm{F} 4 / 80^{+}$population in spleens from properdin-deficient (PropKO) and wildtype (PropWT) mice. Tumour cell suspensions were analysed for the proportion of $\mathrm{CD} 45^{+} \mathrm{CD} 11 \mathrm{~b}^{+} \mathrm{Gr}-1^{+}$cells (a) and splenic suspensions for the proportions of CD $45^{+} \mathrm{CD} 11 \mathrm{~b}^{+} \mathrm{Gr}-1^{+}$cells (b) and of CD206 ${ }^{+} \mathrm{F} 4 / 80+$ cells $(\mathrm{c})$. The data are presented as means \pm SEM. Statistical analysis was performed by unpaired $t$-test. ${ }^{*} p<0.05,{ }^{* *} p<0.01,{ }^{* * *} p<0.001$.

Taken together, this study shows that, whilst properdin deficiency did not alter tumour growth at 14 days post implantation, it significantly diminished inflammatory mediators in serum and tumours as well as immunosuppressive cell populations in the tumour and the periphery. 


\section{Discussion}

Using B16 F10 cell-conditioned media, we have previously described that bone marrow-derived macrophages from properdin-deficient mice were skewed towards an M2 phenotype compared to bone marrow-derived macrophages from wildtype mice [13]. In our in vivo model, however, properdin-deficient mice, when inoculated with logarithmically growing B16F10 cells, showed decreased levels in blood of the chemoattractants $\mathrm{C} 5 \mathrm{a}$ and CCL2 and in tumour of the mediators CCL2, CCL3, IL-13, and TIMP-1. There were significantly fewer M2-skewed macrophages in the spleens of tumour-bearing properdindeficient mice.

We have previously described the complement system to exert measurable effects, acting as adjuvant mediator of anti-tumour immune surveillance, adjuvant promoter of tumour growth, and as factor in tumour immunoediting [15]. In syngeneic tumour mouse models, the general, consistent, observation is that inhibition or blockade of complement is favourable to limiting solid tumour growth over an experimental duration of $12-42$ days $[9,11,12,16,17]$.

C5a is important in the production of the chemoattractant CCL2, as in vitro and in vivo experiments have previously shown $[18,19]$. The origin of elevated levels of circulating CCL2 is likely to be tumour derived, as our study finds significantly elevated levels of CCL2 in tumour lysates prepared from wildtype mice. CCL2 may have autocrine and paracrine, tumour promoting, effects [20]. A mouse model of laser-induced choroidal neovascularisation demonstrated that complement activation and C5b-9 formation precedes the production of CCL2 [21]. In our study, the greater levels of C5a in tumour-bearing wildtype mice were commensurate with elevated CCL2 levels in this group. In the presence of properdin, complement activation has a greater turnover and produces enhanced levels of activation (C5a) and endproducts (C5b-9) when stimulated. CCL2 has recently been shown to be involved in tumour progression, where the genetic absence of CCL2 inhibited recruitment of splenic myeloid-derived suppressor cells immune cells and reduced tumour growth in a syngeneic model [22].

CCL2, CCL3, TIMP-1, and IL-13 are products readily found in an immune response that is driven by M2 macrophages [23], which correspond to a tumour growth-promoting phenotype that aids tumour cell proliferation, reduction of an antigen-specific immune responses, and increased angiogenesis. M2 type macrophages have been shown to induce the differentiation of regulatory $\mathrm{T}$ cells and co-determine the progression of numerous cancers [24]. Analysis of the systemic compartment (spleen) revealed a significant reduction of $\mathrm{M} 2$ type $\left(\mathrm{CD}^{2} \mathrm{6}^{+}\right)$macrophages in tumour-bearing properdin-deficient mice compared to wildtype mice. We propose that properdin deficiency diverted a poorly immunogenic tumour into an "immunologically cold" one [25]. B16F10 cells do not express properdin mRNA (own data and [3]), thus it can be concluded that suppression is due to systemically circulating properdin rather than tumour-produced properdin. However, as complement has a "tonic role" in cell homoeostasis [26] and complement components and receptors are expressed by immune and non-immune cells [27], a possibility that has not been addressed in any of the studies of syngeneic tumour models is that cell activity, in addition to cell recruitment, may differ in the absence (targeted or genetic) of complement [28].

MDSCs, which we found elevated in the spleens and tumours of properdin wildtype mice, can suppress anti-tumour immunity indirectly. Like M2 tumour-associated macrophages, MDSCs generate the cytokines IL-10 and TGF- $\beta$ that can suppress antitumour tumour-infiltrating leukocytes, generate regulatory $\mathrm{T}$ cells in tumour, and convert dendritic cells into a regulatory phenotype. MDSCs may also recruit T-regs to tumours in a TGF- $\beta$-independent pathway [29].

We recognize that there are limitations to this work, which is largely descriptive and employs only one C57BL/6 syngeneic model system for evaluating the effect of properdin on tumour growth. However, our findings have important implications for the role of properdin in modulating the tumour microenvironment, which may be important for 
therapy. A combination approach employing properdin blockade and checkpoint blockade inhibition may be an attractive strategy and warrants further evaluation.

Taken together, our in vivo data corroborate our published in vitro data. They indicate that properdin expression may promote an immunosuppressive tumour microenvironment in part by increasing the expression of chemoattractants (C5a and CCL2) known to recruit MDSCs and M2 macrophages. Combination strategies that include targeting the properdin pathway may be useful in the immunotherapy of melanoma. It is of interest that nucleic acid aptamers have been developed for C5a as well as CCL2 and are in clinical trials [30].

\section{Conclusions}

Properdin is a significant contributor to levels of C5a and CCL2 that are produced in this murine model of melanoma and may play a role in orchestrating immunosuppressive cells in the tumour microenvironment and periphery.

Supplementary Materials: The following are available online at https:/ /www.mdpi.com/1010-660 X/57/2/85/s1: Figure S1: CD45+ CD11b+ Gr-1+ cells from wild type and KO-derived splenocytes; Figure S2: Cytokine array analysis.

Author Contributions: Conceptualization: C.M.S.; methodology and validation: I.A.M.A.-R., L.R.M., and C.M.S.; investigation: I.A.M.A.-R.; formal analysis: I.A.M.A.-R. and C.M.S.; visualization: I.A.M.A.-R.; supervision: L.R.M. and C.M.S.; writing, review, and editing: I.A.M.A.-R., L.R.M., and C.M.S. All authors have read and agreed to the published version of the manuscript.

Funding: This work was supported by a scholarship to I.A.M.A.-R. from The Higher Committee for Education Development in Iraq to study the role of properdin in tumour growth and cell recruitment.

Institutional Review Board Statement: The work was conducted with autority of project license PPL 80/2354 granted by the Secretary of State under the Animals (Scientific Procedures) Act 1986; the amendment to include bioluminescnece protocol was approved 1 December 2014.

Informed Consent Statement: Not applicable.

Data Availability Statement: Data will be shared on request and is contained in PhD thesis AlRayahi, I. A.M. 2017. Role of properdin in tumour growth and cell recruitment. University of Leicester. ISNI 0000000463487063.

Acknowledgments: Staff at the Preclinical Research Facility, where work was conducted, are acknowledged for their husbandry care and support of the project. Hayley Smith (Division of Biomedical Services, University of Leicester) is gratefully acknowledged for her project-related support. We thank M. Browning (Department of Immunology, University Hospitals Leicester NHS Trust, Leicester) for constructive discussions and his encouragement over many years.

Conflicts of Interest: The authors have no conflict of interest to declare.

\section{References}

1. Day, C.P.; Merlino, G.; Van Dyke, T. Preclinical mouse cancer models: A maze of opportunities and challenges. Cell 2015, 163, 39-53. [CrossRef] [PubMed]

2. Dunn, G.P.; Old, L.J.; Schreiber, R.D. The three Es of cancer immunoediting. Annu. Rev. Immunol. 2004, 22, 329-360. [CrossRef] [PubMed]

3. Yu, J.W.; Bhattacharya, S.; Yanamandra, N.; Kilian, D.; Shi, H.; Yadavilli, S.; Katlinskaya, Y.; Kaczynski, H.; Conner, M.; Benson, W.; et al. Tumor-immune profiling of murine syngeneic tumor models as a framework to guide mechanistic studies and predict therapy response in distinct tumor microenvironments. PLoS ONE. 2018, 13, e0206223. [CrossRef] [PubMed]

4. Lechner, M.G.; Karimi, S.S.; Barry-Holson, K.; Angell, T.E.; Murphy, K.A.; Church, C.H.; Ohlfest, J.R.; Hu, P.; Epstein, A.L. Immunogenicity of murine solid tumor models as a defining feature of in vivo behavior and response to immunotherapy. J. Immunother. 2013, 36, 477-489. [CrossRef] [PubMed]

5. Salehen, N.; Stover, C. The role of complement in the success of vaccination with conjugated vs. unconjugated polysaccharide antigen. Vaccine 2008, 26, 451-459. [CrossRef]

6. Bronte, V.; Pittet, M.J. The spleen in local and systemic regulation of immunity. Immunity 2013, 39, 806-818. [CrossRef]

7. Cortez-Retamozo, V.; Etzrodt, M.; Newton, A.; Rauch, P.J.; Chudnovskiy, A.; Berger, C.; Ryan, R.J.; Iwamoto, Y.; Marinelli, B.; Gorbatov, R.; et al. Origins of tumor-associated macrophages and neutrophils. Proc. Natl. Acad. Sci. USA 2012, 109, 2491-2496. [CrossRef] 
8. Afshar-Kharghan, V. The role of the complement system in cancer. J. Clin Investig. 2017, 127, 780-789. [CrossRef]

9. Markiewski, M.; DeAngelis, R.; Benencia, F.; Ricklin-Lichtsteiner, S.; Koutoulaki, A.; Gerard, C.; Coukos, G.; Lambris, J. Modulation of the antitumor immune response by complement. Nat. Immunol. 2008, 9, 1225-1235. [CrossRef]

10. Wang, Y.; Sun, S.N.; Liu, Q.; Yu, Y.Y.; Guo, J.; Wang, K.; Xing, B.C.; Zheng, Q.F.; Campa, M.J.; Patz, E.F., Jr.; et al. Autocrine Complement Inhibits IL10-Dependent T-cell-Mediated Antitumor Immunity to Promote Tumor Progression. Cancer Discov. 2016, 6, 1022-1035. [CrossRef]

11. Nabizadeh, J.A.; Manthey, H.D.; Steyn, F.J.; Chen, W.; Widiapradja, A.; Md Akhir, F.N.; Boyle, G.M.; Taylor, S.M.; Woodruff, T.M.; Rolfe, B.E. The Complement C3a Receptor Contributes to Melanoma Tumorigenesis by Inhibiting Neutrophil and CD4+ T Cell Responses. J. Immunol. 2016, 196, 4783-4792. [CrossRef] [PubMed]

12. Bulla, R.; Tripodo, C.; Rami, D.; Ling, G.S.; Agostinis, C.; Guarnotta, C.; Zorzet, S.; Durigutto, P.; Botto, M.; Tedesco, F. C1q acts in the tumour microenvironment as a cancer-promoting factor independently of complement activation. Nat. Commun. 2016, 7, 10346. [CrossRef] [PubMed]

13. Al-Rayahi, I.A.; Browning, M.; Stover, C. Tumour cell conditioned medium reveals greater M2 skewing of macrophages in the absence of properdin: Properdin shapes macrophage activity in tumours. Immun. Inflamm. Dis. 2017, 5, 68-77. [CrossRef] [PubMed]

14. Stover, C.M.; Luckett, J.C.; Echtenacher, B.; Dupont, A.; Figgitt, S.E.; Brown, J.; Maennel, D.N.; Schwaeble, W.J. Properdin plays a protective role in polymicrobial septic peritonitis. J. Immunol. 2008, 180, 3313-3318. [CrossRef] [PubMed]

15. Stover, C. Dual role of complement in tumour growth and metastasis. Int. J. Mol. Med. 2010, 25, 307-313. [CrossRef]

16. Varela, J.; Imai, M.; Atkinson, C.; Ohta, R.; Rapisardo, M.; Tomlinson, S. Modulation of protective T cell immunity by complement inhibitor expression on tumor cells. Cancer Res. 2008, 68, 6734-6742. [CrossRef]

17. Corrales, L.; Ajona, D.; Rafail, S.; Lasarte, J.J.; Riezu-Boj, J.I.; Lambris, J.D.; Rouzaut, A.; Pajares, M.J.; Montuenga, L.M.; Pio, R. Anaphylatoxin C5a creates a favourable microenvironment for lung cancer progression. J. Immunol. 2012, 189, 4674-4683. [CrossRef]

18. Izumi, S.; Hirai, K.; Miyamasu, M.; Takahashi, Y.; Misaki, Y.; Takaishi, T.; Morita, Y.; Matsushima, K.; Ida, N.; Nakamura, H.; et al. Expression and regulation of monocyte chemoattractant protein-1 by human eosinophils. Eur. J. Immunol. 1997, $27,816-824$. [CrossRef]

19. Piao, C.; Cai, L.; Qiu, S.; Jia, L.; Song, W.; Du, J. Complement 5a Enhances Hepatic Metastases of Colon Cancer via Monocyte Chemoattractant Protein-1-mediated Inflammatory Cell Infiltration. J. Biol. Chem. 2015, 290, 10667-10676. [CrossRef]

20. Conti, I.; Rollins, B.J. CCL2 (monocyte chemoattractant protein-1) and cancer. Semin. Cancer Biol. 2004, 14, 149-154. [CrossRef]

21. Liu, J.; Jha, P.; Lyzogubov, V.V.; Tytarenko, R.G.; Bora, N.S.; Bora, P.S. Relationship between complement membrane attack complex, chemokine (C-C motif) ligand 2 (CCL2) and vascular endothelial growth factor in mouse model of laser-induced choroidal neovascularization. J. Biol. Chem. 2011, 286, 20991-21001. [CrossRef] [PubMed]

22. Li, M.; Knight, D.A.; A Snyder, L.; Smyth, M.J.; Stewart, T.J. A role for CCL2 in both tumor progression and immunosurveillance. Oncoimmunology 2013, 2, e25474. [CrossRef] [PubMed]

23. Sunderkötter, C.; Steinbrink, K.; Goebeler, M.; Bhardwaj, R.; Sorg, C. Macrophages and angiogenesis. J. Leukoc. Biol. 1994, 55, 410-422. [CrossRef] [PubMed]

24. Sun, W.; Wei, F.Q.; Li, W.J.; Wei, J.W.; Zhong, H.; Wen, Y.H.; Lei, W.B.; Chen, L.; Li, H.; Lin, H.Q.; et al. A positive-feedback loop between tumour infiltrating activated Treg cells and type 2-skewed macrophages is essential for progression of laryngeal squamous cell carcinoma. Br. J. Cancer 2017, 117, 1631-1643. [CrossRef] [PubMed]

25. Mosely, S.I.; Prime, J.E.; Sainson, R.C.; Koopmann, J.O.; Wang, D.Y.; Greenawalt, D.M.; Ahdesmaki, M.J.; Leyland, R.; Mullins, S.; Pacelli, L.; et al. Rational Selection of Syngeneic Preclinical Tumor Models for Immunotherapeutic Drug Discovery. Cancer Immunol. Res. 2017, 5, 29-41. [CrossRef]

26. Ricklin, D.; Hajishengallis, G.; Yang, K.; Lambris, J.D. Complement: A key system for immune surveillance and homeostasis. Nat. Immunol. 2010, 11, 785-797. [CrossRef]

27. Lubbers, R.; van Essen, M.F.; van Kooten, C.; Trouw, L.A. Production of complement components by cells of the immune system. Clin. Exp. Immunol. 2017, 188, 183-194. [CrossRef]

28. Reis, E.S.; Mastellos, D.C.; Ricklin, D.; Mantovani, A.; Lambris, J.D. Complement in cancer: Untangling an intricate relationship. Nat. Rev. Immunol. 2018, 18, 5-18. [CrossRef]

29. Serafini, P.; Mgebroff, S.; Noonan, K.; Borrello, I. Myeloid-derived suppressor cells promote cross-tolerance in B-cell lymphoma by expanding regulatory T cells. Cancer Res. 2008, 68, 5439-5449. [CrossRef] [PubMed]

30. Zhang, Y.; Lai, B.S.; Juhas, M. Recent Advances in Aptamer Discovery and Applications. Molecules 2019, 24, 941. [CrossRef] 